\title{
Joachim Becker
}

\section{Oligarchie - eine Form bürgerlicher Herrschaft Das Beispiel osteuropäischer semi-peripherer Kapitalismus}

Oligarchie ist in Lateinamerika ein vielfach benützter Begriff. Allerdings wird er dort zur Charakterisierung der Herrschaftsverhältnisse heute etwas weniger bemüht als in der Vergangenheit. Dafür erlebt er andernorts eine Renaissance nämlich in Europa, speziell in dessen östlicher Peripherie. In Westeuropa ist die Forza Italia als eine von einem Medienmogul und im Immobiliengeschäft groß gewordenen Unternehmer gegründete und wie ein Unternehmen geführte Partei ein exemplarischer Fall einer oligarchischen Formation. In Österreich sind bei den letzten Nationalratswahlen gleich zwei oligarchische Parteien - das Team Stronach, das allerdings bereits im fortgeschrittenen Zerfallsprozess übergangen ist, sowie die Neos mit einer dezenteren oligarchischen Komponente - eingezogen. Auffällig ist, dass in Westeuropa oligarchische Formationen speziell dort auftreten, wo das Bürgertum traditionell auf Schwierigkeiten stieß, eine hegemoniale Rolle wahrzunehmen, und bürgerliche Herrschaft sich in der fordistischen Zeit stark auf klientelistische Formen der Herrschaftssicherung stützte. In den Ländern der osteuropäischen (Semi-)Peripherie ist ein Bürgertum erst in den letzten 25 Jahren erneut geschaffen worden. Hier sind teils sehr ausgeprägte oligarchische Strukturen im Rahmen der kapitalistischen Transformation entstanden. Daher bieten sich gerade osteuropäische Staaten für eine nähere empirische Untersuchung oligarchischer Herrschaftsformen an.

\section{Oligarchische Herrschaftsformen und die aktuelle kritische Staatsdiskussion}

Zentrales Kennzeichen oligarchischer Herrschaft ist die besonders enge Verschränkung von ökonomischer und politischer Macht (vgl. van der Pijl 2015: 48, 51ff.). Dies impliziert, dass Kapitalgruppen einen relativ direkten Zugriff auf den Staatsapparat haben. Unter Bezug auf Poulantzas (2002: 159), ließe sich also festhalten, dass der Grad der Autonomie eines derartigen (semi-)peripheren Staates gegenüber der Fraktion einheimischer Großkapitalisten, möglicherweise aber auch gegenüber Einzelkapitalen mithin relativ gering ist. Wenn die Interessen 
der dominanten oligarchischen Gruppen auseinanderlaufen, stellt der direkte Durchgriff von Einzelkapitalen auf staatliche Entscheidungszentren nicht nur die Kohärenz der staatlichen Politik, sondern potenziell auch die Kohäsion des Staates infrage. Ein zentraler Transmissionsriemen zwischen Staatsbürgern und Staatsapparat und Zugangsmechanismus zum Parlament als einer wichtigen staatlichen Arena stellen Parteien dar. Hier fragt sich, wie oligarchische Gruppen Parteien kontrollieren und ob Parteienkonkurrenz durch oligarchische Konkurrenz geprägt ist.

Oligarchische Strukturen weisen auf eine relative Schwäche der Zivilgesellschaft als Feld der Interessenformierung, Normbildung und Interessendurchsetzung hin, wie sie vielfach für peripher-kapitalistische Staaten charakteristisch ist (Becker 2008a: 20). Das heißt allerdings nicht, dass zivilgesellschaftliche Strukturen für oligarchische Herrschaft irrelevant sind. Über zivilgesellschaftliche Strukturen können dominante Kräfte ihre Herrschaft über Elemente des Konsenses, der Normbildung und der zumindest selektiven Kooptierung abstützen. Insofern stellt sich speziell die Frage, inwieweit oligarchische Gruppen Institutionen der Normbildung, wie beispielsweise Medien, kontrollieren und wie sie auf oppositionelle soziale Bewegungen reagieren.

Eine (semi-)periphere Rolle im globalen Kontext hat allerdings nicht nur politische, sondern auch ökonomische Strukturen Konsequenzen. Die Rolle des Auslandskapitals ist vielfach beträchtlich. Das Verhältnis von Auslandskapital und einheimischer (gelegentlich aber auch teils internationalisierter) Oligarchie ist durch Elemente von Kooperation und Konkurrenz geprägt, wobei, abhängig von der jeweiligen Konjunktur, mal das eine und mal das andere Element an Bedeutung gewinnt. Für eine Einschätzung der ökonomischen und politischen Macht der Oligarchie ist auf jeden Fall wichtig, wie stark konzentriert sie ist, welche Branchen sie kontrolliert und wie ihr Verhältnis zum Auslandskapital ist. Der Staat stellt hierbei einen potenziellen Schutzmechanismus gegenüber dem Auslandskapital dar. Auf jeden Fall sind die ökonomischen Machtpositionen der einheimischen Oligarchie stark unmittelbar politisch fundiert (vgl. van der Pijl 2015: 55).

Bei der konzeptionellen Fassung und Analyse oligarchischer Herrschaftsformen liegt der Fokus auf dem Nationalstaat, dem einheimischen Kapital und dem Klassencharakter des Staates - und damit auf Bereichen, die in der aktuellen kritischen Staatsdiskussion im deutschsprachigen Bereich und auch generell in Westeuropa eher vernachlässigt werden. Diese setzt die Akzente primär auf der Mehrebenenanalyse der EU-Staatlichkeit - mit besonderer Betonung der europäischen Ebene. Das gilt beispielsweise für Hauke Brunkhorst (2014), der den von Marx geprägten Begriff des Bonapartismus bei der Analyse der EUStaatsstrukturen bemüht und die These einer Veränderung von innen vertritt. 
Marx hebt in seiner Bonapartismus-Analyse nicht nur die scheinbare Verselbständigung des Staates und die besonders hervorgehobene und eigenständige Rolle der Exekutive, sondern auch die instabile Klassenkonstellation hervor (Marx 1852: 123f.). Während in der starken Rolle exekutiver Staatsorgane Parallelen zur aktuellen Diskussion gesehen werden können, unterscheidet sich der Charakter der Kräfteverhältnisse grundlegend von der aktuellen Situation in Europa, gerade auch in der EU. Die bürgerliche Dominanz tritt unverhüllt zutage. Selbst minimale Kompromissbereitschaft haben die EU-Organe gegenüber der griechischen Syriza-Regierung, die Positionen vertritt, die der westeuropäischen Sozialdemokratie der 1970er Jahre entsprechen, und eine sozialere Ausgestaltung der Austeritätspolitik verlangte, nicht gezeigt. Stattdessen wischte der EU-Gipfel am 12./13. Juli 2015 das griechische Referendum gegen die Austerität beiseite und herrschte der Syriza-Regierung ein radikal rechtsliberales Strukturanpassungsprogramm auf. Die Lesart einer bonapartistischen Konstellation überzeugt bezogen auf die EU nicht. Oligarchische Herrschaftsformen, die deutlich auf nationalstaatlicher Ebene beobachtet werden können, haben als Fusion politischer und ökonomischer Macht individueller Kapitalgruppen bzw. Kapitalisten keinen bonapartistischen Charakter.

Überzeugender ist die Diagnose einer signifikanten Stärkung exekutiver Staatsstrukturen und Marginalisierung parlamentarisch-demokratischer Verfahren in der Mehrebenen-Staatlichkeit der EU, wie sie nicht nur von Brunkhorst, sondern beispielsweise auch von Oberndorfer (2012a; b) und Vauchez (2014) herausgearbeitet werden. Oberndorfer (2012b: 417ff.) hebt hierbei speziell die Institutionalisierung von Regelbindungen der Wirtschaftspolitik in unterschiedlichen rechtlichen Arrangements hervor, durch die parlamentarische Instanzen an bestimmte Regeln dauerhaft gebunden werden und Exekutivorgane Definitionsmacht über die Auslegung der Regeln erhalten. Unter Bezugnahme auf Poulantzas' Begriff des „autoritären Etatismus“ (Poulantzas 2002: 231ff.), der in Hinblick auf den spät-fordistischen Staat entwickelt worden war, benennt Oberndorfer (2012a; b) dieses Herrschaftsdispositiv als „,autoritären Wettbewerbsetatismus". Er verweist zudem auf die konzeptionell-theoretischen Wurzeln der grundlegenden Konzepte in autoritären Liberalismus-Konzeptionen der späten Weimarer Republik. Vauchez (2014) akzentuiert hingegen in seiner Analyse der EU-Staatlichkeit die zentrale Rolle der Technokratie und von demokratischer Kontrolle und Legitimierung „unabhängiger“ Institutionen, wie der Europäischen Zentralbank oder den Europäischen Gerichtshof. Diese Formen autoritärer Staatlichkeit können zwar bei oligarchischer Herrschaft eine Rolle spielen, sind aber nicht deren Kern. Bei oligarchischer Herrschaft geht es gerade auch um die Kontrolle einzelner Kapitalgruppen über diskretionäre staatliche Entscheidungen, die für die jeweilige Unternehmenspolitik relevant sind. 
Weniger auf die EU-Institutionen fokussiert ist Crouchs Konzept einer „Postdemokratie“. Als Kernelemente einer Postdemokratie identifiziert Crouch einerseits die Reduzierung von Wahlkämpfen auf die Schlacht konkurrierender PR-Teams, welche eine spezifische Form der Definition von Problemen durchsetzen könnten, und die damit verbundene Transformation von Wahlkämpfen zu einem „reinen Spektakel“ und andererseits die reale Aushandlung staatlicher Politik zwischen gewählten Regierungen und „Eliten, die vor allem die Interessen der Wirtschaft vertreten." (Crouch 2008: 10). Bei den Wirtschaftsinteressen stellt Crouch (ebd.: 45ff.) primär auf „globale Unternehmen“ ab, während einheimische Kapitalgruppen, die allerdings in der Regel durchaus eine Internationalisierungspolitik verfolgen, den Kern oligarchischer Herrschaftsstrukturen stellen. Bei der von ihm beobachteten Transformation von Parteien stellt Crouch (ebd.: 93ff.) vor allem die Emanzipation der Parteiführungen von der - immer kleiner werdenden - Mitgliedschaft und den starken Bedeutungsgewinn von Berater- und Lobbyistenzirkeln für die Definition der Parteipolitik ab. Die Parteienfinanzierung geschehe vor allem über mit den jeweiligen Parteien eng verbundenen Unternehmen. Eine besonders reine Form habe der Typ der postdemokratischen Partei in Berluscionis Forza Italia angenommen (ebd.: 96ff.). Explizit als oligarchische Partei diskutiert Crouch die Forza Italia allerdings nicht. Als ein zentrales Interessenfeld für Wirtschaftsinteressen, die zunehmend an Einfluss gewinnen, identifiziert Crouch (ebd.: 103ff.) die Kommerzialisierung öffentlicher Dienstleistungen. Dies ist auch tatsächlich ein zentrales Betätigungsfeld des politisch gut verbundenen einheimischen Großkapitals in Osteuropa. Oligarchische Herrschaftsformen schreiben sich allerdings - wenngleich in einer sehr spezifischen Art und Weise - in von Crouchs Konzept der Postdemokratie Transformationen der Staatlichkeit ein. Allerdings entstanden die osteuropäischen Formen oligarchischer Herrschaft nicht, wie in Westeuropa, aus dem Fordismus, sondern aus dem Staatssozialismus heraus.

Diese Verbindung von einer zweiten grundlegenden Phase ursprünglichen Akkumulation und Herausbildung oligarchischer Strukturen lässt Mechanismen der Genese oligarchischer Herrschaftsformen in Osteuropa besonders deutlich hervortreten. Besonders deutlich wird das in den Fällen, in denen neue Nationalstaaten aus zerfallenden multi-ethnischen Staaten entstanden. Daher sollen osteuropäische Nachfolgestaaten der Sowjetunion, Jugoslawiens und der Tschechoslowakei im Kern der folgenden Analysen stehen. In allen diesen (kon-) föderalen Staaten war das Bestreben auf Ebene der in einzelnen Teilrepubliken politischen dominanten Kräfte nach Kontrolle des Privatisierungsprozesses eine zentrale Triebkraft des Staatszerfalls. Gleichzeitig weisen die Nachfolgestaaten der Sowjetunion, Jugoslawiens und der Tschechoslowakei bezogen auf ihre Stellung in der internationalen Arbeitsteilung, auf die sich herausbildenden Eigentums-, 
Sozial- und Staatsstrukturen auch signifikante Unterschiede auf. Die Analyse der Herausbildung oligarchischer Strukturen im Kontext staatlicher Desintegration scheint auch im Hinblick auf eine durchaus mögliche (zumindest partielle) Desintegration der EU besonders interessant. Als Fallbeispiele wird die Herausbildung oligarchischer Strukturen in ausgewählten Nachfolgestaaten der Sowjetunion (Russland und Ukraine), Jugoslawiens (Serbien) und der Tschechoslowakei (Tschechische Republik und Slowakei) vergleichend untersucht. Der Vergleich stellt drei Leitfragen in den Vordergrund:

Wie ist die einheimische Oligarchie entstanden?

Wie hat sich ihr Verhältnis zum semi-peripheren Staat entwickelt und wie ist hierbei ihr Verhältnis zu politischen Parteien als zentralen Transmissionsriemen zu staatlichen Entscheidungszentren?

In welchen ökonomischen Sektoren haben sich die oligarchischen Gruppen etablieren können?

\section{Russland: vom Oligarchenregime zur Schaffung einer stärker autonomen Staatlichkeit}

Gebahnt wurde der Weg zu einer kapitalistischen Transformation Osteuropas und damit einer neuen grundlegenden Phase ursprünglicher Akkumulation in der Region durch die polit-ökonomischen Prozesse in der späten Sowjetunion. Im Rahmen der Perestroika, die eine Reaktion auf ökonomische Stagnationstendenzen und ein relatives Zurückfallen gegenüber den kapitalistischen Kernländern darstellte, standen sich unterschiedliche gesellschaftspolitische Konzeptionen gegenüber. Diese reichten von Kräften der Beharrung auf den überkommenen Strukturen, über ParteigängerInnen einer grundlegenden, aber graduellen Veränderung staatssozialistischer Strukturen, einschließlich Schritten der Demokratisierung und der Schaffung eines marktlichen Teilbereichs, bis hin zu AnhängerInnen einer kapitalistischen Transformation (Ivanter 2012: 33f.). Als zentrale gesellschaftliche Kraft für die Privatisierung und die Durchsetzung einer kapitalistischen Transformation sieht Dzarasov (2014: 63ff.) im Management der Staatsbetriebe, das in der späten Sowjetunion zunehmend an Spielräumen gewann. Dem privatisierungsfreundlichen Block rechnet er aber auch eine Gruppe von (wirtschafts-)liberal orientierten Intellektuellen und ÖkonomInnen zu. Diese gewannen vornehmlich auf der Ebene einiger sowjetischer Teilrepubliken politische Verbündete in den Partei- und Staatsstrukturen. Von besonderer Bedeutung war hier die Unterstützung aus Reihen der russischen Partei- und Staatsstrukturen. Boris Jelzin wurde zum Exponenten der radikaltransformatorischen Kräfte (Jaitner 2014: 38). Die gradualistischen Reformkräfte hingegen besaßen Rückhalt 
in Teilen der „sowjetischen Wirtschafts- und Verwaltungselite“ (Ivanter 2012: 34), obgleich auch auf Unionsebene die radikalen Transformationsbefürworter an Terrain gewannen.

Ein signifikanter Teil des radikal privatisierungsfreundlichen Lagers in Russland sah die Unionsstrukturen zunehmend als Hindernis für seine Transformationsstrategie wahr und stellten den Erhalt der Sowjetunion infrage (Jaitner 2014: 39f.). Nachdem es einen maßgeblichen Anteil am Scheitern des Putschversuchs gegen den damaligen sowjetischen Parteivorsitzenden Michail Gorbatschow im August 1991 geleistet hatte, gewann dieses Lager entscheidende Machtpositionen und leitete die Auflösung der Sowjetunion ein (ebd.: 52ff.). Doch auch nach der Auflösung der Sowjetunion hatten die auf eine rasche und radikale Transformation setzenden Kräfte noch nicht alle institutionellen Widerstände ausgestaltet. Das radikale Privatisierungsprogramm von 1992 stieß sowohl in der Bevölkerung als auch im Kongress der Volksdeputierten auf Ablehnung (Nekipelov 2012: 78). Parlamentarisch umfasste die Opposition ein breites Spektrum von Kräften - ihr Minimalkonsens richtete sich auf eine Stärkung der inländischen Produktion, inländischer Unternehmer und sozialer Abfederung. Im Herbst 1993 eskalierte der Konflikt und das Jelzin-Lager schaltete das Parlament unter Einsatz militärischer Gewalt aus. Eine neue Verfassung zentralisierte Machtbefugnisse beim Präsidenten. Zur Durchsetzung seiner radikalen Transformationsstrategie, die mit einem fast ein Jahrzehnt andauernden schweren wirtschaftlichen Ein- oder präziser Zusammenbruch einherging, etablierte das Jelzin-Regime einen „, neuen Autoritarismus" (Jaitner 2014: 92).

Wie Nekipelov (2012: 78) unterstreicht, setzte die Jelzin-Regierung politisch darauf, „die Bildung einer spezifischen sozialen Schicht zu begünstigen, welche die Unterstützung ihrer Politik garantierte." Diese neu geschaffene „Schicht“ waren die OligarchInnen. Sie wurden gezielt durch die Ausgestaltung der Privatisierungspolitik geschaffen, die die kapitalistischen Radikaltransformatoren für eine Zementierung der kapitalistischen Umgestaltung als zentral ansahen. Kern der Privatisierungspolitik von 1992 bis 1994 war die sogenannte VoucherPrivatisierung, also die Vergabe von Anteilsscheinen. Begünstigt waren formal die Belegschaften beim Erwerb der Anteilsscheine, real aber das jeweilige Management, in dessen Händen die Anteilsscheine dann auch oft landeten. Auch die wenigen Insider, die zu einer Einschätzung der jeweiligen Firmenwerte, in der Lage waren, wurden durch die Voucher-Privatisierung begünstigt. Formal war die Voucher-Privatisierung, die als „Volksprivatisierung“ politisch vermarktet wurde, breit angelegt. Real resultierte sie in einer starken Eigentumskonzentration (Jaitner 2014: 72f.). Zudem ermöglichte die Regierung die Herausbildung eines privaten Bankensektors, der sich auf den Handel mit Devisen und Staatspapieren konzentrierte. Die neuen Privatbanken stellten vielfach den Kern der 
Firmenimperien der sich herausbildenden Gruppe von OligarchInnen dar. Einen großen Sprung bei der Ausweitung dieser um Banken herum gruppierten Firmenimperien stellte das 1995 aufgelegte Programm von Pfandauktionen dar. Der Staat nahm im großen Stil Kredite gegen die Stellung von Sicherheiten in Form lukrativer Firmenbeteiligungen für den Fall, dass Zahlungen nicht pünktlich geleistet würden, auf. Die als Sicherheit gestellten Aktiva waren vielfach grotesk unterbewertet. Auf diese Art fanden sehr lukrative Firmenbeteiligungen im Rohstoffsektor ihren Weg in Oligarchenhände (u.a. Nekipelov 2012: 79f.; Jaitner 2014: 110ff.). Die entstehenden großen Kapitalgruppen hatten als Kern Banken bzw. Finanzgruppen und Rohstoffaktivitäten, weshalb sie Stykow (2007) als „Finanz-Industrie-Gruppen“ charakterisiert. Zudem legten sie sich Medienhäuser $\mathrm{zu}$, was ihnen eine Beeinflussung der Meinungsbildung eröffnete.

Die OligarchInnen wiederum stützten das Jelzin-Regime und die sie tragende Hauptpartei, die wechselnden Namen hatte, aber von Holzer (1999: 167) von ihrer Funktion her als „Partei der Macht“ gekennzeichnet wird. Massive Medienkampagnen zugunsten waren eine zentrale Form oligarchischer Unterstützung für das Präsidentenlager.

Die Oligarchenstrukturen wurden 1998 - noch in der Regierungszeit Jelzins durch die schwere Finanzkrise Russlands erschüttert. Der private Bankensektor, Kern verschiedener Kapitalgruppen, wurde durch die Krise erheblich geschwächt, Oligarchengruppen mit einem starken Rohstoffschwerpunkt überstanden die Krise deutlich besser (Macek 2015: 121). Die Preishausse bei den internationalen Rohstoffpreisen stimulierte in den Folgejahren die russische Ökonomie und ließ die Staatseinnahmen sprudeln, die starke Abwertung des Rubels in Gefolge der Wirtschaftskrise ermöglichte aber auch eine Stabilisierung der industriellen Produktion. Der Aufschwung der 2000er Jahre war mithin nicht allein rohstoffgetragen, sondern ruhte auf einer breiteren Grundlage. Er erlaubte breiten Bevölkerungsgruppen eine Verbesserung und Stabilisierung ihrer sozialen Lage. Dies war auch die primäre Legitimierungsressource der auf die Jelzin-Ära folgenden Putin-Medwedew-Regierungen. Verwundbarkeiten durch die internationale Rohstoffkonjunktur und im Bankensektor blieben jedoch bestehen - und wurden mit der globalen Krise 2008/2009 auch schlagend (vgl. Becker/Dziwulska 2010).

Die Krise von 1998 hatte aber auch politische Folgen. Der kurzzeitig amtierende Premierminister Jewgeni Primakow setzte in der Anti-Krisen-Politik stärker bewusst staatliche Regulierung institutionell stabilisierende Elemente. Mit seiner Orientierung auf den Aufbau eines Entwicklungsstaates vermochte sich Primakow nicht durchzusetzen, aber in den dominanten Kräften setzte ein Umdenken ein, „demzufolge erst staatliche Kontrolle und Organisation eine funktionierende Wirtschaft garantieren "(Jaitner 2014: 141). Repräsentant einer Neuausrichtung auf eine stärkere strategische Rolle des Staates in der russischen 
kapitalistischen Ökonomie und der entsprechenden Begrenzung des politischen Durchgriffs einzelner Oligarchengruppen auf den Staat wurde Wladimir Putin, der auf Jelzin als Staatspräsident folgte. Im Unterschied zu Primakow war Putin weniger auf Re-Industrialisierung und die Schaffung eines Entwicklungsstaates orientiert. Insofern war er für die dominanten Oligarchengruppen mit ihrer starken Orientierung auf den Rohstoffsektor akzeptabler als Primakow.

Aber auch Putin, dessen engeres Umfeld liberale juridische und ökonomische Technokratengruppen, aber auch der Sicherheitsapparat darstellten, veränderte das Verhältnis von Staat und Oligarchengruppen. Die Regierung Putin drängte den Einfluss individueller Oligarchengruppen auf staatliche Strukturen zurück, baute den staatlichen Einfluss im Öl- und Gassektor aus und erhöhte die Besteuerung, wiederum speziell im Rohstoffsektor (Pirani 2010: 70). Gegen einzelne politisch stark exponierte OligarchInnen ging die Staatsmacht scharf vor. Besonders emblematisch war hierfür der Fall Chodorkowski. Michail Chodorkowski hatte eine typische Oligarchen-Karriere der Jelzin-Zeit hinter sich: Über seine Komsomol-Funktionärstätigkeit zu Sowjetzeiten gut verbunden erlangte Chodorkowski über sein Engagement im Bankensektor zu großem Vermögen - und letztlich zu einer Schlüsselstellung im Ölsektor (vgl. Dzarasov 2014: 68). Die neuen Spielregeln der Putin-Regierung - staatliche Leitlinien im Öl- und Gasgeschäft sowie politische Zurückhaltung - akzeptierte er nicht. Er verlor nicht nur sein Firmenimperium, sondern wurde auch wegen gravierender Steuervergehen verurteilt (vgl. Pirani 2010: 71 ff.).

Während der Staat im Gassektor über Gazprom auch in der Jelzin-Zeit stark präsent blieb, weitete die Regierung Putin den Staatsanteil im Ölsektor bis zum Jahr 2007 von einem Viertel auf Zweidrittel aus (Macek 2015: 123). Damit ist die russische Regierung im Energiesektor zu einer strategischen Politik in der Lage, privates Kapital - in- wie ausländischer Herkunft - ist hier in einer untergeordneten Funktion. Oligarchiegruppen sind in Schlüsselbranchen - gerade auch im Rohstoffbereich - präsent geblieben, genießen dort eine informelle Form staatlicher Konzessionen (ebd.: 116f.). Der Staat zielt auf eine gezielte Balance zwischen verschiedenen Wirtschafts- und Oligarchengruppen. Diesen wurde im Rahmen dieses Neuarrangements die Konsolidierung ihres in der Jelzin-Ära angeeigneten Firmenbesitzes ermöglicht. Ein Element der Instabilität in den Eigentumsstrukturen blieb aber bestehen und ist eine der Ursachen für ein eher kurzfristig angelegtes Investitionsverhalten (Dzarasov 2014).

Hand in Hand mit der Zentralisierung der Staatsmacht gingen die Konsolidierung der „Partei der Macht“, jetzt Jedinaja Rossija („Einiges Russland“), sowie die Unterordnung und Kooptierung gesellschaftlicher Organisationen. Die Regierung-Putin setzte den eigenständigen Medienimperien und brachte die Medien weitgehend auf Linie (Pirani 2010: 69ff.; Macek 2015: 110). 
Mithin hat die Regierung Putin die Autonomie des Staates gegenüber individuellen Oligarchengruppen entscheidend gestärkt. Als Klassenfraktion bleiben die großen russischen Kapitalgruppen hingegen für die Regierung Putin in zentralen wirtschaftspolitischen Fragen handlungsleitend. Dies wurde beispielsweise deutlich, als der Verfall der Rohstoffpreise und Sanktionen den Rubel 2014 auf Talfahrt schickten. Eine für die Stabilisierung des Rubelkurses naheliegende Reaktion wäre angesichts der starken Kapitalabflüsse, die 2014 von der russischen Zentralbank auf $150 \mathrm{Mrd}$. US-Dollar geschätzt wurden (OSW 2015: 12), die Einführung von Kapitalverkehrskontrollen gewesen. Diese wurde aber - im Interesse der Oligarchengruppen - nicht einmal in Erwägung gezogen. Die stärker durch den Rückgang der Öl- und Gaspreise denn durch die im Rahmen des Ukrainekonflikts verhängten westlichen Sanktionen verursachte Verschlechterung der wirtschaftlichen Situation erodiert die zentrale Legitimationsressource der etatistisch-oligarchischen Ordnung - die im Vergleich zum Chaos und Niedergang der Jelzin-Jahre erreichte Stabilisierung der Lebensverhältnisse. Als Kompensation gilt zunehmend ein nationalistischer Diskurs, der durch den Konflikt in der Ukraine und die zunehmend scharfe Frontstellung der meisten westlichen Länder gegenüber Russland einen starken Aufschwung der erfahren hat. Wie Boris Kargarlitski (2014: 88) hervorhebt, stehen die mit der Konfrontation mit den westlichen Ländern einhergehenden Erfordernisse einer stärkeren Binnenorientierung der Wirtschaft, einer stärkeren Kooperation mit den BRICS-Ländern und veränderte internationaler Regeln in einem Spannungsfeld zu den ökonomischen Interessen der russischen OligarchInnen.

\section{Ukraine: von der Oligarchenkonkurrenz zum drohenden Staatszerfall}

Eine der Haupttriebkräfte hinter der Unabhängigkeit der Ukraine waren hohe Kader der ukrainischen Sektion der KPdSU - auch ihnen ging es um einen direkten Einfluss auf den Transformations- und Privatisierungsprozess (vgl. Duleba 1998: 27 ff.). Wie auch in Russland gehen die Anfänge der Privatisierung in der Ukraine bis in spät-sowjetische Zeit Ende der 1980er Jahre zurück. Die Privatisierungen wurden in der Regel so gestaltet, dass politische Insider zum Zug kamen (Yurchenko 2012: 132). In der Amtszeit des zweiten Staatspräsidenten der Ukraine, Leonid Kutschma (1994-2005), in der zumindest eine gewisse Konsolidierung der Staatsstrukturen erfolgte, genoss das einheimische Kapital eindeutig den Vorrang (Bojcun 2011: 154). Partiell abhängig vom Standort variieren die Schwerpunkte der Konglomerate der entstehenden Oligarchengruppen etwas: Für die Ostukraine ist die Schwerindustrie bedeutsamer, vielfach sind 
Teile der Energiewirtschaft und auch Banken integriert (vgl. Bojcun 2011: 154 ff.; Yurchenko 2012: 133 ff.; Matuszak 2012: 8, 13ff., 49ff.; Leshchenko 2014). Die ukrainischen Medien werden weitgehend von den OligarchInnen kontrolliert (Matuszak 2012: 35ff.).

In den 1990er Jahren kristallisierten sich bereits die beiden wichtigsten regionalen Oligarchiezentren in Dnipropetrowsk und Donetzk (oder breiter: dem Donbass) heraus. Erstere rekrutierte sich vornehmlich aus Kadern der ukrainischen Kommunistischen Partei und war anfänglich besonders eng mit dem zweiten Staatspräsidenten der unabhängigen Ukraine, Leonid Kutschma, verbunden. $\mathrm{Zu}$ den ersten Öl- und Gas-OligarchInnen der Ukraine zählten Pawlo Lasarenko und Julija Tymoschenko, die anfänglichen Aufstieg der Patronage Kutschmas verdankten, zeitweilig politische Spitzenpositionen wahrnahmen und beide später Gefängnisstrafen abbüßen mussten (Lazarenko wegen Geldwäsche in den USA, Tymoschenko wurde erstmals 2001 wegen Steuervergehen inhaftiert und war ein zweites Mal von 2011 bis 2014 im Zusammenhang mit Gasgeschäften mit Russland im Gefängnis).

Im Unterschied zu Russland geht es beim ukrainischen Gas- und Ölgeschäft vornehmlich um ein Import- und Transportgeschäft. Konkurrierend zum Dnipropetrowsker Kapital entstand das Donetzker Kapital, das in seiner Entstehung eng mit dem organisierten Verbrechen verbunden war. Für die Donetzker Kapitalgruppen ist die Schwerindustrie von zentraler Bedeutung für ihre Firmenimperien. Die schwerindustrielle Produktion ist sehr energieabhängig. Dies war einer der Faktoren, warum die beiden regionalen Hauptoligarchenfraktionen um die Kontrolle des Energiesektors rangen. Während der Regierungszeit Kutschmas gewann allmählich die Donetzker Gruppe an Einfluss. Kutschmas Kandidat für seine Nachfolge, Wiktor Janukowytsch, kam aus dem Donetzker Umfeld (Yurchenko 2012: 132 ff.). Die Donetzker Gruppe wies auch eine stärkere Kohäsion auf als die Dnipropetrowsker OligarchInnen.

Bereits sehr früh gewannen die OligarchInnen entscheidenden Einfluss auf Parteien, die zur Konsolidierung der politischen Einflusskanäle dienten. Daher charakterisiert der polnische Sozialwissenschaftler Sławomir Matuszak (2012) die politische Ordnung der Ukraine auch als „oligarchische Demokratie“. Die Abgrenzungslinien zwischen den Parteien liefen primär anhand der Betonung unterschiedlicher Nationskonzepte - stark ethno-nationalistisch mit teils expliziten Bezügen auf faschistische Nationalistengruppen der 1930er/1940er Jahre in der Westukraine, eher territorial und die ukrainischen wie russischen Elemente betonend im Osten des Landes (vgl. Studenna-Skrukwa 2014: 77ff., 89). Gegen Ende der zweiten Amtszeit Kutschmas repräsentierte die Partei der Regionen in besonderem Maße das Donetzker Großkapital. Ihr Präsidentschaftskandidat war 2004 Wiktor Janukowytsch. Gegen Janukowytsch stellten sich nicht nur 
Naša Ukraina von Wiktor Juschtschenko, der eher als Repräsentant des mittleren Kapitals galt (Matuszak 2012: 20), sondern andere Oligarchengruppen, gerade auch aus Dnipropetrowsk. Die Anti-Janukowytsch-Allianz hatte aufgrund ihrer explizit pro-westlichen Ausrichtung auch die Unterstützung aus den USA und europäischen Ländern, während sowohl Kutschma als auch Janukowytsch außenpolitisch auf ein Lavieren zwischen USA/EU und Russland setzten. Damit erhielt die OligarchInnen-Konkurrenz eine internationale Dimension.

Gegen OligarchInnen-Herrschaft und autoritäre Tendenzen gab es in der zweiten Amtszeit Kutschmas bereits Proteste. Diese Protestwelle erhielt angesichts von Wahlfälschungen zugunsten von Janukowytsch bei der Präsidentschaftswahl von 2004 einen starken Schub - und wurde auch aus westlichen Ländern stark unterstützt (Becker 2006). Die Proteste waren erfolgreich - Juschtschenko wurde Ende 2004 in einer wiederholten Stichwahl zum neuen Präsidenten gewählt.

Diese Ereignisse werden oft als „orangene Revolution“ bezeichnet. Eine Revolution war es jedoch nicht. Vielmehr wurden die Plätze an der Sonne neu verteilt (vgl. Segert 2015: 23; Müller 2015: 140 ff.), aber auch das Donezker Kapital konnte wichtige Positionen bewahren. In der durch ein kreditgetriebenes Wachstum, das bis 2008 aber auch nur 70 Prozent des BIP-Niveaus von 1989 erreichte (vgl. Myant/Drahokoupil 2011: Tab A2), und beschleunigte Privatisierung gekennzeichneten Amtszeit Juschtschenkos konnte das Auslandskapital, gerade auch im Bankensektor, seine Position ausbauen (Bojcun 2011: 162f.). Aber auch die Oligarchengruppen vermochten, ihre Firmenimperien auszuweiten.

Das politische Lager der „orangenen Revolution“ zeigte wenig politische Kohäsion und zerrieb sich in inneren politischen Konflikten. Dies vermochten die Partei der Regionen und das hinter ihr stehende Donetzker Kapital für politische Terraingewinne zu nutzen. Zeitweise kam es zu einer Kohabitation zwischen Juschtschenko und Janukowytsch. Nachdem infolge der internationalen Krise die ukrainischen Exporte stark eingebrochen, der Bankensektor ins Schleudern geraten und das BIP 2009 um fast 15 Prozent zurückgegangen waren, gewann Janukowytsch die Präsidentschaftswahlen von 2010.

Damit verschoben sich die Kräfteverhältnisse zwischen den Oligarchengruppen erneut. Janukowytsch vermochte einerseits die wirtschaftliche Lage auf sehr niedrigem Niveau zu stabilisieren; andererseits förderte er auf eine Weise das Firmenimperium seines engsten familiären Umfeldes, wie es selbst in der äußerst rabiaten (Selbst-)Bereicherungspolitik der unabhängigen Ukraine noch nicht der Fall gewesen war (vgl. Matuszak 2012: 41ff.; Parafianowicz/Potocki 2015: Kap. 2). Damit engte Janukowytsch aber auch seine eigene Machtbasis ein. Diese Politik schuf nicht nur Spannungen mit anderen Oligarchengruppen, sondern verstärkte - gerade angesichts der äußerst schlechten sozialen Lage - auch die Ablehnung in breiten Bevölkerungskreisen. 
Die entscheidende Blöße gab sich Janukowytsch aber mit seiner erratischen und auch widersprüchlichen Außenpolitik. Eigentlich war er - durchaus der doppelten wirtschaftlichen Ausrichtung der Ukraine sowohl auf die EU als auch Russland entsprechend - auf eine Schaukelpolitik zwischen Moskau und Brüssel orientiert. Allerdings wurde in seiner Amtszeit das Assoziationsabkommen mit der EU, das zur Übernahme eines großen Teils des EU-Rechtsbestandes verpflichtet und damit eng und einseitig an die EU bindet (Becker 2014), fertig verhandelt. Die Gründe hierfür sind nicht ganz klar. Gestützt auf zahlreiche Interviews mit ukrainischen und EU-Offiziellen führen die polnischen Journalisten Zbigniew Parafianowicz und Michał Potocki (2015: 128f.) als Motive die Absicherung der Wiederwahl im Jahr 2015 durch Stärkung der Wählerbasis im Westen und Zentrum des Landes, die Absicherung der Resultate der ursprünglichen Akkumulation sowie den Schutz gegenüber russischer Konkurrenz in bestimmten Bereichen an. Allerdings waren das Regierungslager und die hinter ihm stehenden Wirtschaftsgruppen in der Frage gespalten (ebd.: 185). Angesichts des russischen Drucks und der - anscheinend sehr spät realisierten - extrem hohen Kosten des Assoziationsabkommens rückte Janukowytsch kurz vor dessen geplanter Unterzeichnung von einem Abschluss ab.

Für die gut gebildeten GroßstädterInnen, vor allem im Zentrum und Westen des Landes, schwand damit die - allerdings illusionäre - Aussicht, über das EUAssoziationsabkommen Schritte in Richtung Rechtsstaatlichkeit und stärkere Prosperität zu machen. Sie gingen auf die Straße. Kaum Resonanz hatten die Proteste im eher auf Russland orientierten Osten des Landes. Angesichts der Repression seitens der Sicherheitskräfte erhielten die Proteste in einer zweiten Welle eine starke Stoßrichtung gegen die Regierung. Zwar richteten sich die Proteste auch gegen die oligarchischen Strukturen, aber einige zentrale OligarchInnen, wie beispielsweise der heutige Petro Poroschenko, unterstützten die Proteste und auch die oppositionellen, sich pro-westlich gebenden Oligarchenparteien wie auch die faschistische Swoboda waren Teil der Protestbewegung. Diese erhielt Unterstützung aus westlichen Ländern (Kronauer 2014: 146 ff.), ein Teil der Oligarchengruppen, speziell aus Dnipropetrowsk, hatte langjährige enge politische Verbindungen in die USA (Paulsen 2014: 9). Insofern überlagerten sich Anti-OligarchInnen-Protest und internationalisierte Konflikte zwischen Oligarchengruppen ähnlich wie während der „orangenen Revolution“. Es gab bei den Protesten Ende 2013/Anfang 2014 aber auch einen gravierenden Unterschied zu 2004: Das Auftreten paramilitärischer Gruppen rechtsextremer Provenienz, die der Protestbewegung ein partiell sehr militantes, paramilitärisches Gepräge gaben und deren ultra-nationalistischen Parolen auf zunehmende Akzeptanz stießen (vgl. u.a. Ishchenko 2014; Heyden 2015: 13ff., 62ff.). Angesichts der starken und militanten Protestwelle, der passiven Polizei (außer den Sondereinheiten) und seiner schrumpfenden Machtbasis zeigte 
die Regierung Janukowytsch Anfang 2014 deutliche Auflösungserscheinungen (Müller 2015: 144). Ein geordneter Abtritt, der unter internationaler Vermittlung eigentlich ausgehandelt worden war, kam letztlich nicht mehr zustande, die Regierung Janukowytsch wurde im Februar 2014 gestürzt.

Der Regierungswechsel hatte außenpolitisch und für die innenpolitische Konstellation weit schärfere Konsequenzen als 2014. Die neue Regierung ging schärfer noch als Juschtschenko 2005 auf Westkurs. Innere regionale Spaltungslinien verschärfte das Regierungslager durch ein rasch im Parlament verabschiedetes (dann allerdings nicht in Kraft gesetztes) Sprachengesetz, das sich gegen die russische Sprache richtete und symbolisch hoch aufgeladen war. Damit leisteten sie der von Russland massiv unterstützten Separationsbestrebungen der Krim und erst Föderalisierungs-, dann Separationsforderungen in Teilen der Ostukraine, vor allem im Donbass, Vorschub. Letztere wurden zumindest anfänglich von Teilen des Donetzker Kapitals, speziell dem reichsten ukrainischen Kapitalisten Rinat Achmetow, unterstützt, aber nicht kontrolliert (Plank 2014: 85). Russland ist in die militärische Destabilisierung der Ukraine involviert. Teile des Donbass sind unter faktischer Kontrolle von Rebellenkräften, die auch Rudimente eigener Staatsstrukturen geschaffen haben.

Die Paramilitarisierung ist aber nicht auf die regierungsfeindlichen Kräfte in der Ostukraine beschränkt, sondern auch im Regierungslager erkennbar. So finanziert beispielsweise der Oligarch Ihor Kolomojskyj (aus Dnipropetrowsk), Eigentümer der größten ukrainischen Bank und zeitweilig von der Regierung eingesetzter Gouverneur von Dnipropetrowsk, stark rechtsextrem eingefärbte paramilitärische Einheiten. Diese fanden anscheinend nicht nur bei militärischen Operationen in der Ostukraine, sondern auch bei Ausweitung von Kolomojskyjs Firmenimperium Einsatz (Schuller 2015: 5). Im März 2015 wurde Kolomojskyj nach einem Auftritt seiner Einheiten im Konflikt um die Kontrolle des staatlichen Ölkonzerns zum Rücktritt von seinem Gouverneursposten gezwungen. Der US-Botschafter vermochte offenbar in einem vorhergehenden Gespräch mit Kolomojskyj dem Rücktrittsansinnen - vermutlich mit Hinweisen auf dessen Vermögenssituation - den nötigen Nachdruck zu verleihen (Heyden 2015: 170f.).

Die Dominanz der OligarchInnen in der Ukraine ist ungebrochen. Dies zeigt auch die erstmalige Wahl eines der wichtigsten OligarchInnen, nämlich des Waffen- und Schokoladenindustriellen Petro Poroschenko, zum Präsidenten an. Die Kräfteverhältnisse zwischen den Oligarchengruppen haben sich allerdings deutlich verschoben. In der Tendenz zeichnen sich laut Konończuk (2015: 8) eine Schwächung des schwerindustriellen und ein Aufstieg des agroindustriellen Kapitals ab. Diese Tendenz dürfte durch das Assoziationsabkommen mit der EU, das den Konkurrenzdruck auf die Schwerindustrie erhöht, verfestigt werden. Das Kapital aus dem Donbass ist durch den politischen Umschwung und den Krieg 
in der Ostukraine signifikant geschwächt, aber weder ökonomisch noch politisch ausgeschaltet worden. Die Kräfte aus dem Lager der früheren Partei der Regionen haben sich parteipolitisch neu gruppiert und sind im Oktober 2014 neu gewählten Parlament vertreten, wenn auch deutlich schwächer (Pogorzelski 2015a). Deutlich gestärkt sind andere Oligarchengruppen - Kolomojskij und Dmytro Firtasch (allerdings mit Problemen mit der US-Justiz)/Sergei Lavochkin - aus den Wahlen hervorgegangen. Ihre VertreterInnen sind in mehreren Parteien stark vertreten - es gilt die Strategie der politischen Einflussdiversifizierung (Konończuk 2015: 6f.). Im Regierungslager sind zwar auch politische AktivistInnen aus der Protestbewegung über die Parteien ins Parlament gekommen, aber auch sie gestehen den fortgesetzten oligarchischen Charakter der Parteien ein (Pogorzelski 2015b).

Im Kontext eines beschleunigten ökonomischen Niedergangs ist der Konflikt zwischen den Oligarchengruppen soweit eskaliert, dass der ukrainische Staat kein legitimes Gewaltmonopol mehr besetzt. In Teilen des Landes ist er nicht legitim - und er hat aufgrund der Existenz sowohl, wenn auch prekär, dem Regierungslager zuzurechnenden als regierungsfeindlichen paramilitärischen Kräften kein Gewaltmonopol mehr. Seine ökonomischen Handlungsspielräume sind aufgrund der starken Krise gering, der Druck des Internationalen Währungsfonds (IWF) ist stark. Die staatlichen Gegenstrukturen in Teilen des Donbass sind noch schwächer. Hier spielen paramilitärische Gruppen eine starke Rolle. Die Hochburgen der politischen Kräfte in der Ukraine sind regional polarisiert. Die inneren Konflikte werden durch den geopolitischen Konflikt zwischen USA/EU einerseits und Russland andererseits überlagert. Angesichts dieser Lage werden bereits eine „Bosnisierung der Ukraine“ und ein „neues Dayton“ angedacht (Danas 2015: 9). Dies würde die faktische Aufteilung des Landes in Kantone bedeuten - im Fall der Ukraine wären diese stark auf die jeweiligen außenpolitischen Partner orientiert. Im Gegensatz zu Bosnien-Herzegowina, das ein faktisches internationales Protektorat ist, würde die Ukraine wohl zwei innere Protektorate mit einer schwachen Zentralregierung aufweisen. Aber auch andere Szenarien sind denkbar. Erkennbar ist, dass die eskalierte Oligarchenkonkurrenz, die durch internationale Bündnisbeziehungen in geo-politische Konkurrenzkämpfe eingegliedert worden ist, die Staatlichkeit der Ukraine extrem geschwächt hat und zu einem katastrophalen Verlauf der ökonomischen wie sozialen Entwicklung des Landes beigetragen hat.

\section{Serbien: Kriegsgewinnler und Tajkuni}

Im post-jugoslawischen Raum haben OligarchInnen bzw. politisch gut verbundenes einheimisches Großkapital, in der Region als Tajkuni bezeichnet, ihre 
Wurzeln vielfach im Auflösungsprozess Jugoslawiens und den damit verbundenen Kriegen. Das Streben der politisch dominanten Kräfte in den einzelnen Republiken war ein zentraler Faktor beim Zerfall Jugoslawiens (Samary 2005: 57). Die darauf folgende Entstehung der Tajkuni stand im engen Zusammenhang mit den jeweiligen Machtzentren (Antonić 2006: 27f.; Malenica 2007: 133f.; Zgodić2011: 94ff.). Wo, wie im Fall Bosnien-Herzegowina, ethnische Quoten im Rahmen international vermittelter Kompromissabkommen zum zentralen Merkmal der Staatsstruktur wurden, verfestigten diese auch oligarchische Eigentums- und Einfluss-Strukturen auf ethnischer Basis (Mujkić 2007: XIIIf.). Im Fall militärischer Konflikte erfolgte die ursprüngliche Akkumulation unter besonders intransparenten Bedingungen. Die im post-jugoslawischen Raum entstandenen großen einheimischen Kapitalgruppen sind in der Regel Mischkonzerne. Den Kern des Unternehmensimperiums Agrokor des reichsten Unternehmers der Region, dem Kroaten Ivica Todorić, bilden Handelsunternehmen in verschiedenen Nachfolgestaaten Jugoslawiens (vgl. Marković 2015).

In das für den (post-)jugoslawischen Raum typische Grundmuster des Aufstiegs der Tajkuni fügt sich auch Serbien ein. Die Privatisierungspolitik der SlobodanMilošević-Regierungen, deren Kern die aus dem Bund der Kommunisten Serbiens hervorgegangene Socijalistička partija Serbije (SPS) bildete, erfolgte unter den sehr spezifischen Bedingungen von Krieg und westlichen Sanktionen (vgl. Musić 2013: 17ff.; 2014: 376ff.), die einerseits Intransparenz förderten und andererseits die Arbeiterschaft atomisierten. Die Anfang der 1990er Jahre entstehende neue „ökonomische Elite“, wie Lazić (2011: 74) sie nennt, hatte ihre Ursprünge damals zu etwa Zweidritteln in der Nomenklatura des Staatssozialismus. Hierbei gab es Prozesse einer De-Facto- und De-Jure-Privatisierung. Hohe Staatskader - beispielsweise Minister, die gleichzeitig Direktoren strategischer Staatsunternehmen waren - vermochten Staatseigentum und - unter Sanktionsbedingungen - staatliche Importmonopole zur eigenen Bereicherung zu nutzen. Teils legten sie die Gewinne in Immobilien an (vgl. Antonić 2002: 374). Die formale Privatisierung begünstigte Insider - vom Gesetz her sowohl Belegschaften als auch Manager. Real gewannen Manager im Regelfall rasch die Kontrolle über die Unternehmen (Stojiljković 2013: 247, Musić 2014: 376f.). Importbegünstigungen und privilegierter Zugang zu Krediten der überwiegend noch staatlichen Banken waren zentrale Kanäle für das Wachstum einheimischer, regierungsnaher Kapitalisten - wie beispielsweise auch heute noch bedeutsame Tajkuni wie Miroslav Mišković (Delta-Gruppe) oder Milan Beko (vgl. Antonić 2002: 371ff.). Die internationale Isolation des Milošević-Regimes war allerdings gleichzeitig auch eine Akkumulationsgrenze für die neue serbische Bourgeoisie, gerade auch die großen Tajkuni. Diese orientierten sich daher auf die pro-westliche Opposition um und unterstützten auch im Jahr 2000 deren Wahlkampagne (Lazić 2011: 65, Musić 
2014: 380). Massendemonstrationen, die sich an Wahlfälschungsvorwürfen entzündet hatten, führten im Oktober 2000 zum Sturz der Regierung Milošević und zu einer Regierungsübernahme der überwiegend neo-liberal orientierten Oppositionsparteien, die die Unterstützung der westlichen Länder genossen.

Die neo-liberalen Regierungen nach dem Jahr 2000 beschleunigten die Privatisierungspolitik. Schüsselbereiche, vor allem der Bankensektor, gingen an das Auslandskapital (vgl. Uvalic 2010: 150ff.). Aber auch die Tajkuni konnten ihre Firmenimperien ausbauen. Bei einigen großen Privatisierungen zugunsten der Tajkuni, oftmals im Bereich von Infrastrukturunternehmen und -konzessionen, hegt die EU-Kommission Korruptionsverdacht (vgl. Stojiljković 2013: 258ff.). Die Privatisierungspolitik ab dem Jahr 2001 hat zu einer sehr hohen Eigentumskonzentration bei den ,neuen Oligarchen“ und Auslandsunternehmen geführt, resümiert Uvalic (2010: 208). Laut Antonić (2006: 36f.) wies in den Jahren nach 2001 ein Teil der einheimischen Tajkuni, wie z.B. Mišković, Bindungen zum Auslandskapital auf und stützte gemeinsam mit diesem die stark außenorientierte neoliberale Politik. Die Verbindungen zwischen Tajkuni und den jeweiligen Regierungsparteien blieben eng (Mladenovic 2014: 14; vgl. etwa den journalistischen Bericht von Bačević 2012). Im Medienbereich sind die OligarchInnen - wie übrigens auch der serbische Staat - deutlich präsent, die Berichterstattung ist außerordentlich konformistisch (vgl. Antonič 2006: 98f., Cohen/Lampe 2011: 209ff.).

Im Jahr 2012 kam es insofern zu einer partiellen politischen Zäsur, als eine Koalition der Srpska napredna stranka (SNS), die aus dem nationalistischen Lager kommt, sich aber eine Pro-EU-Orientierung zugelegt hat, SPS sowie kleineren Parteien an die Regierung kam. Ihr führendes Personal hatte teils bereits in der Milošević-Ära Karriere gemacht. Bereits kurz nach der Regierungsbildung demonstrierte der Vorsitzende der SNS und starke Mann der Regierung, Aleksandar Vučić, Distanz zu einzelnen OligarchInnen, wie etwa Miroslav Mišković (vgl. das Interview mit Nikčevi 2012). Diese Distanzierung war allerdings eher selektiv, denn auf der SNS-Liste befand sich mit Karić auch ein sehr bekannter Oligarch (Ranković 2013: 28). Ende desselben Jahres wurde allerdings Mišković, einer der wichtigsten Oligarchen des Landes, wegen Wirtschaftsvergehen in Untersuchungshaft genommen. Diese Maßnahme war in der serbischen Bevölkerung extrem populär. Bei vorgezogenen Neuwahlen Anfang 2014 kam die SNS fast auf 50 Prozent der Stimmen (Stojiljković 2014: 25).

Das Vorbild für die SNS scheint die Putin-Regierung in Russland zu sein. Allerdings war die Disziplinierung der OligarchInnen in Serbien weniger einschneidend. Wirtschaftlich setzt die Regierung Vučić stark auf große Immobilienprojekte in Belgrad - speziell „Beograd na vodi“(Vilenica et al. 2015, Rudić 2015). Hierfür hofft sie auf Investoren aus der arabischen Halbinsel, aber solche Projekte wären - im Falle ihrer Realisierung - auch ein Eldorado für die OligarchInnen. 
Tschechische Republik und Slowakei: Oligarchische Elemente in extern dominierten Eigentumsstrukturen

Bei der 1992 ausgehandelten Trennung der Tschechoslowakei in Tschechische Republik und Slowakei ging es auch um die Ausrichtung der Transformationspolitik und die Kontrolle der Privatisierungsprozesse. Sowohl die von Václav Klaus (Občanská demokratická strana, ODS) geführte tschechische wie die von Vladimír Mečiar (Hnutie za demokratické Slovensko, HZDS) geführte slowakische Regierung waren bestrebt, jeweils eine „nationale Bourgeoisie“ zu schaffen. Allerdings verfolgten sie hierbei unterschiedliche Wege: In der Tschechischen Republik stand zunächst eine Coupon-Privatisierung im Vordergrund, die noch in der tschechoslowakischen Zeit begonnen worden war. Diese Privatisierungsmethode wurde als eine Art Volkskapitalismus propagiert. Schnell sammelten sich die interessanten Kapitalbeteiligungen in einigen wenigen Finanzfonds, vielfach der Kern der späteren einheimischen Bourgeoisie. Zunehmend gewannen Direktverkäufe an Individuen, die als „dynamische Unternehmer“ (Myant/ Drahokoupil 2011: 242) eingeschätzt wurden (vgl. allgemeiner Myant 2003; Mertlík 1999). In der Slowakei waren Direktverkäufe vorherrschend, die das Management der bisherigen Staatsbetriebe begünstigte (Hallon/Sabol 2013: 404). In beiden Fällen stützte der zunächst noch staatliche Bankensektor die neuen einheimischen Kapitalisten mit Krediten. Im Kern des Interesses standen in den 1990er Jahren die Industriebetriebe. Allerdings stellte sich die Umstellung der Betriebe von binnenmarktorientierter bzw. auf die Arbeitsteilung der staatssozialistischen Länder orientierter Produktion auf Westimporte als schwierig dar. Eine systematische unterstützende Industriepolitik gab es nicht. Daher gewannen breit diversifizierte Finanzholdings bereits in den 1990er Jahren an Bedeutung (Brzica 2006).

Bis Ende der 1990er Jahre stand in beiden Ländern der Versuch im Vordergrund, eine einheimische Bourgeoisie zu schaffen. Die einheimischen Kapitalisten hatten oftmals enge Verbindungen in die regierenden Parteien, vor allem ODS und HZDS. Als oligarchische Parteien können diese trotzdem nicht qualifiziert werden. Ende der 1990er Jahre änderte sich die politische Konstellation. Mit dem Beginn der Beitrittsgespräche mit der EU stieg der Druck, zugunsten der westeuropäischen Konzerne zu privatisieren. In der Tschechischen Republik wurde die ODS durch Korruptionsskandale und eine Bankenkrise geschwächt und verlor die Regierung an die Sozialdemokratie. In der Slowakei wurden der HZDS von der liberalen Opposition, die massiv von US-amerikanischen Stiftungen unterstützt wurde, autoritäre Tendenzen vorgeworfen. Auch sie verlor 1998 die Wahlen gegen ein breites Oppositionsbündnis. 
In beiden Ländern erfolgte in die Folgezeit eine Privatisierungspolitik primär zugunsten des Auslandskapitals. Dieses gewann die Kontrolle über den Bankensektor und große Teil der Industrie - und damit über die Schlüsselsektoren des abhängigen Entwicklungsmodells (vgl. Becker 2008b: 7ff.). Die forcierte Privatisierungspolitik eröffnete aber auch den einheimischen Kapitalgruppen - meist in der Form von Finanzholdings - Möglichkeiten zur Expansion. Dies galt gerade auch für Bereiche der materiellen oder sozialen Infrastruktur (vgl. Hallon/Sabol 2013: 437). Teils boten die Gruppen allein, teils - speziell bei besonders großen Vorhaben - in Konsortien mit Auslandsunternehmen. Für den Fall der Slowakei halten Hallon und Sabol (2013: 407) fest, dass der Verkauf von strategischen Infrastrukturen, aber auch anderen Bereichen „oft unter ihrem Wert realisiert wurde und mit Klientelismus verbunden war." Zeitweise gewann beispielsweise die Penta-Gruppe starken Einfluss auf das slowakische Gesundheitsministerium. Dies begünstigte ihre Strategie, sich in der gesamten Gesundheitskette von der Übernahme von Kliniken bis hin zur Krankenversicherung festzusetzen.

In den letzten Jahren haben die einheimischen großen Kapitalgruppen sowohl in der Tschechischen Republik (vgl. Tabery 2014; Mikulka 2015) als auch in der Slowakei (vgl. Čobejová/Múčka 2014) ihren Einfluss auf den Mediensektor ausgebaut. Einige dieser Übernahmen haben erhebliche öffentliche Kontroversen ausgelöst. Das gilt speziell für den Kauf der beiden wichtigsten liberal-konservativen Tageszeitungen, MF Dnes und Lidové Noviny, durch den Großindustriellen Andrej Babiš, nachdem dieser seine Partei ANO gegründet hatte und die politische Bühne betreten hatte.

Die Partei ANO ist das bislang erfolgreichste oligarchische Parteienprojekt in den beiden Ländern. ANO erzielte bei den Parlamentswahlen 2013 18,6 Prozent der Stimmen und wurde damit zweitstärkste Kraft im Parlament. In einer Koalition zusammen mit Sozial- und Christdemokraten wurde Babiš Finanzminister - und erhielt damit den wirtschaftspolitischen Schlüsselposten. Babiš, dessen Agrofert-Gruppe vor allem in der Agro- und Chemieindustrie aktiv ist, war zuvor politisch breit verbunden, einen wichtigen Ausschlag für die Parteigründung, so Pergler (2014: 130f.) in seiner Babiš-Biografie, dürfte die Verärgerung über ihn direkt betreffende wirtschaftspolitische Entscheidungen (Biotreibstoffe) gewesen sein. Wirtschaftspolitisch ist er weniger scharf rechts orientiert als die anderen liberal-konservativen Parteien. Er stellte seine Partei als Anti-Establishment-Kraft dar, die effizient geführt werde (vgl. Uherek 2013). Babiš lernte aus dem Beispiel des Aufstiegs und raschen Falls von Věci veřejné (VV), die 2010 ins Parlament gewählt wurden und ebenfalls Koalitionspartei wurde (Pergler 2014: 131). VV hatte bereits gezeigt, wie leicht es angesichts der Unzufriedenheit mit der sozialen Lage und den zahlreichen Skandalen war, mit Anti-Korruptionsparolen ins Parlament zu kommen. VV, die letztlich aus einer 
privaten Sicherheitsagentur heraus gegründet worden war und erfolgreich das Innenministerium angestrebt hatte, zeigte sich allerdings der politischen Praxis nicht gewachsen. Diese geht Babiš professioneller an. In der Slowakei gelangte eine erste oligarchische Formation, ebenfalls ANO abgekürzt, bereits 2002 ins Parlament und zu ersten Regierungsehren. Kleinere oligarchische Parteien wurden auch später in der Slowakei gegründet. Hier wurde 2014 mit Andrej Kiska ein Geschäftsmann, der aus dem Finanzsektor kam, als „unabhängiger“ Kandidat der politischen Rechten zum Präsidenten gewählt (vgl. Rybár et al. 2014). Oligarchische Tendenzen in der Politik sind mithin in beiden Ländern erkennbar.

In der Tschechischen Republik haben die oligarchischen Formationen seit 2010 den Erosionsprozess der traditionellen tschechischen Rechtsparteien, speziell der ODS, zumindest teilweise kompensiert und der politischen Rechten entweder die Regierungsbildung (2010) oder doch zumindest die Regierungsbeteiligung (2013) ermöglicht. Tendenziell haben sich die oligarchischen Parteien mit ihren schwachen Organisationsstrukturen als wenig dauerhaft erwiesen. $\mathrm{Ob}$ die tschechische ANO eine Ausnahme darstellen wird, ist noch nicht absehbar. Sie sind nicht das dominante Parteienmodell in den beiden zentralosteuropäischen Ländern. Die Bindungen zwischen einheimischem Kapital und Parteien sind allerdings insgesamt (mit Ausnahme der Kommunistischen Partei) eng.

\section{Oligarchien im regionalen Vergleich}

In Osteuropa wurde Kapitalismus zunächst ohne Kapitalisten geschaffen (vgl. Eyal et al. 1998). Aspiranten auf eine Kapitalisten-Position gab es hingegen schon - vor allem unter den damaligen Direktoren der Staatsunternehmen, teils auch anderer Kader. Sie konnten diese Position allerdings nur mithilfe des Staatsapparates gewinnen. Der Staat wurde zu einem zentralen Kampffeld um ökonomische Positionen. Hieraus resultierten - zumindest zunächst - sehr enge Bindungen zwischen der neuen einheimischen Bourgeoisie und dem Staat.

In allen analysierten Ländern Osteuropas stand in den 1990er Jahren der Aufbau einer einheimischen Bourgeoisie im Vordergrund. Speziell in den beiden sowjetischen Nachfolgestaaten Russland und Ukraine entstand eine äußerst konzentrierte Eigentumsstruktur. Das einheimische Großkapital hat hier maßgeblichen Einfluss auf ökonomische Schlüsselbranchen nehmen können, allerdings hat der russische Staat nach der Krise von 1998 in zwei Kernsektoren - dem Energie- und dem Bankensektor - strategische Positionen ausbauen bzw. wiedergewinnen können. In Serbien ist das einheimische Kapital ebenfalls hochgradig konzentriert, der Bankensektor ging jedoch in den 1990er Jahre in westeuropäische Hände über. Mit dem Beginn der EU-Beitrittsverhandlungen 
wurden die Tschechische Republik und die Slowakei deutlich enger in die internationalisierte Produktion der deutschen Exportindustrie eingebunden und gingen die beiden ökonomischen Schlüsselsektoren - Banken und Exportindustrie - überwiegend in Hände des Auslandskapitals über. Für die einheimischen großen Kapitalgruppen - überwiegend Finanzholdings - verblieben primär Bereiche der materiellen und sozialen Infrastruktur, der Immobiliensektor und die Bauwirtschaft. Überwiegend haben die großen einheimischen Kapitalgruppen den Charakter von Mischkonzernen. Industriell sind sie vor allem in Branchen mit Rohstoffbasis - z.B. der Schwerindustrie in der Ukraine oder agrobasierte Industrie - präsent. Hierin kommt der semi-periphere Charakter der jeweiligen Ökonomien zum Ausdruck. Medial sind die Oligarchengruppen im Regelfall stark präsent. Ein durchgängiges Charakteristikum ist in der osteuropäischen Region der außerordentlich hohe Grad der Konzentration des einheimischen Kapitals. Die hohe einheimische Kapitalkonzentration wurde durch die Privatisierungspolitik der Regierungen begünstigt, gleichzeitig schaffte der große Geschäftsumfang eine günstige materielle Basis für die dauerhafte politische Einflussnahme der einheimischen großen Kapitalgruppen bzw. ihrer Eigner.

Extrem hoch war die Verzahnung von einheimischem Kapital und dem Staatsapparat in den Nachfolgestaaten der Sowjetunion und Jugoslawiens in den 1990er Jahren. Während der russische und serbische Staatsapparat zumindest eine begrenzte Autonomie gegenüber oligarchischen Einzelkapitalen und gegenüber der Gesamtfraktion des oligarchischen Kapitals gewann, war dies in der Ukraine nicht der Fall. Hier hat sich der Zugriff von oligarchischen Einzelkapitalen auf den Staat nicht gelockert. Ökonomische und politische Konkurrenz von Oligarchengruppen gehen Hand in Hand. Die Oligarchengruppen sind hierbei auch internationale politische Bündnisse eingegangen. Ihr internationalisierter Konflikt stellt den ukrainischen Staat vor eine Zerreißprobe.

In der Tschechischen Republik und der Slowakei pflegen die einheimischen großen Kapitalgruppen enge Kontakte mit den politischen Parteien. Der Erosion der stärker etablierten Rechtsparteien hat, vor allem in der Tschechischen Republik, Raum für oligarchische Parteien geschaffen. Diese pflegen einen Anti-Establishment-Diskurs und streichen die Effizienz ihrer unternehmensähnlichen Strukturen hervor. Hiermit haben sie in der Tschechischen Republik die Abschwächung der ODS als traditioneller Rechtspartei kompensieren und so der politischen Rechten eine Regierungsübernahme oder zumindest -beteiligung ermöglichen können. Oligarchische Parteien zeichnen sich durch eine große Instabilität aus - vielfach sind sie nur eine Legislaturperiode im Parlament vertreten. Hohe Instabilität der oligarchischen Parteien schließt eine hohe Stabilität des oligarchischen Einflusses auf die politische Sphäre keines Falls aus. Oligarchische Regime haben sich durchaus in der Lage gezeigt, gesellschaftliche 
Widersprüche zu bearbeiten und sehr inegalitäre gesellschaftliche Verhältnisse aufrechtzuerhalten. Das zeigt das Beispiel der Regierung Putin. Wenn allerdings die Konkurrenz von Oligarchengruppen, wie in der Ukraine, aus dem Ruder läuft, ist die (oftmals prekäre) Stabilität bedroht. Gleichzeitig schaffen Konflikte zwischen Oligarchenfraktionen nicht unbedingt Spielräume zur Schwächung der Oligarchenherrschaft. So veränderten zwei große Protestwellen, die sich zumindest teilweise gegen Oligarchenherrschaft richteten, nur die Kräfteverhältnisse zwischen Oligarchengruppen, nicht aber die gesellschaftlichen Verhältnisse.

Ansätze oligarchischer Herrschaft und die Herausbildung oligarchischer Parteien lassen sich auch in Westeuropa erkennen. Das gilt speziell dort, wo, wie in Italien, Griechenland oder Österreich die Bourgeoisie sich traditionell mit der Erringung einer hegemonialen Position schwertat und stark auf klientelistische Bindungsmuster zurückgreifen musste. Mit einer Verschärfung der Verteilungskämpfe in strukturell stagnierenden Ökonomien und dem Versuch des Kapitals, sich bisher öffentliche Sphären als Kapitalanlagebereiche anzueignen, ist eine verstärkte oligarchische Tendenz auch in den westeuropäischen Ländern zu erwarten. Ein möglicher, allmählicher Desintegrationsprozess der Eurozone und der EU, wie er mit dem deutschen Vorschlag eines vorübergehenden griechischen Ausscheidens aus der Eurozone und dem Diktat der Euro-Gruppe gegenüber der griechischen Regierung deutlicher näher gerückt ist (vgl. Münchau 2015: 9), könnte der Herausbildung oligarchischer Herrschaftsformen in zumindest einigen Staaten der heutigen EU neue Dynamik verleihen.

\section{Literatur}

Antonić, Slobodan (2002): Zarobljena zemlja. Srbija za vlade Slobodana Miloševića, Belgrad.

- (2006): Elita, građanstvo i slaba država, Belgrad.

Bačević, Batić (2012): Sponzor iz ormara. In: NIN, 20.12: 18-19-

Becker, Joachim (2006): Von Oligarchie zu Oligarchie. Zur politischen Ökonomie der „farbigen“

Revolutionen. In: Kurswechsel, Nr. 1: 117-124.

- (2008a): Der kapitalistische Staat in der Peripherie: polit-ökonomische Perspektiven, in: Journal

für Entwicklungspolitik 24(2): 10-32.

- (2008b): Der Drang nach Osten: Wirtschaftliche Interessen und geopolitische Strategien. In:

Kurswechsel, Nr. 4: 5-29.

- (2014): Untergeordnete Integration: das Assoziationsabkommen zwischen der EU und Ukraine.

In: Kurswechsel, Nr. 3: 76-82.

-/Dziwulska, A. (2010): BRIC Countries in Crisis: Russia and Brazil Compared. In: Mysljaeva,

I.N./Budanov, M.A./Golionko, E.S. (Hg.): Gosudarsvennoe upravlenie v XXI veke: tradicii i innovaci, Moskau: 31-37.

Brunkhorst, Hauke (2014): Das doppelte Gesicht Europas - Zwischen Kapitalismus und Demokratie, Berlin.

Brzica, Daneš (2006): Vývoj firemných skupín ako dôsledok koncentrácie vlastníctva po roku 1993: príklad Českej republiky a Slovenskej republiky, in: Ekonomický časopis, 45(8): 771-784. 
Bojcun, Marko (2011): The Ukrainian Economy and the International Financial Crisis. In: Dale, Gareth (Hg.): First the Transition, then the Crash. Eastern Europe in the 2000s, London: 143-168.

Cohen, Lenard/Lampe, John R. (2011): Embracing Democracy in the Western Balkans. From Postconflict Struggles Toward European Integration, Washington D.C.-Baltimore.

Crouch, Colin (2008): Postdemokratie, Frankfurt/M.

Čobejová, Eva/Múčka, Fero (2014): SME ide o vela. In: Týždeň, 13.10., 4-7.

Danas (2015): Milan Pani: Krim je ruski, in: Danas, 11.-12.4.: 9.

Duleba, Alexander (1998): Koniec súčasnej strednej Európy? Ukrajina a Slovensko po prvej vlne rozšírenia NATO, Bratislava.

Dzarasov, Ruslan (2014): The Conundrum of Russian Capitalism. The Post-Soviet Economy in the World-System, London.

Eyal, Gil/Szelenyi, Iván/Townley, Eleanor (1998): Making Capitalism Without Capitalists. The New Ruling Elites in Eastern Europe, London-New York.

Hallon, Ludovit/Sabol, Miroslav (2013): Proces globalizácie a vstup Slovenska do medzinárodných hospodárskych štruktur po r. 1993. In: Londák, Miroslav/Michálek, Slavomir (Hg.): 20 rokov samostatnej Slovenskej republiky. Jedinečnost a diskontinuita historického vývoja, Bratislava: 397-439.

Heyden, Ulrich (2015): Ein Krieg der Oligarchen. Das Tauziehen um die Ukraine, Köln.

Holzer, Jan (1999): Politické strany Ruska. Hledaní identity, Brno.

Ishchenko, Volodomyr (2014): Ukraine's Fractures. In: New Left Review, New Series 87: 7-33.

Ivanter, Viktor (2012): La transition macroéconomique et politique. In: Sapir, Jacques (Hg.): La transition russe, vingt ans après, Paris: 21-64.

Jaitner, Felix (2014): Einführung des Kapitalismus in Russland. Von Gorbatschow zu Putin, Hamburg. Kagarlitski, Boris (2014): El modelo Putin: de la normalización política a la crisis de Ucrania. In: Nueva Sociedad, Nr. 253: 72-88.

Konończuk, Wojciech (2015): Oligarchowie po Majdanie: stary system na „nowej“ Ukrainie, Komentarze OSW 162, Warschau.

Kronauer, Jörg (2014): Die widersprüchliche Imperative der deutschen Ostpolitik, in: Strutynski, Peter (Hg.): Ein Spiel mit dem Feuer. Die Ukraine, Russland und der Westen, Köln: 137-152.

Lazić, Mladen (2011): Čekajući kapitalizam. Nastanak novih klasnih odnosa u Srbiji, Belgrad.

Leshchenko, Serhii (2014): Hinter den Kulissen. Eine Typologie der ukrainischen Oligarchen. In: Transit, Nr. 45: 102-117.

Macek, Ivan (2015): Zdroje a limity Putinova režimu v Rusku: semikartelizace, koncesní ekonomika a strukturálně-ropná past. In: Veber, Václav/Štorková, Pavla (Hg.): Rusko a Evropa 2013. K 100. výročí vydání Masarykovy knihy Rusko a Evropa, Pardubice: 108-131.

Malenica, Zoran (2007): Ogledi o hrvatskom društvu, Zagreb.

Marković, Radmilo (2015): Forbesova rang-lista najuspešnijih biznesmena: Povratak Miroslava Miškovića. In: Vreme, Nr. 1274, 4.6. URL: vreme.com/cms/view.php?id=1302822\&print=yes, Zugriff: 16.6.2015.

Marx, Karl (1852): Der 18. Brumaire des Louis Bonaparte, Frankfurt/M 1965.

Matuszak, Sławomir (2012): Demokracja oligarchiczna. Wpływ grup biznesowych na ukraińska politykę, Prace OSW 42, Warschau.

Metrlík, Pavel (1999): The Emerging Ownership Structure in Central and Eastern Europe. In: Potůček, Martin (Hg.): Česká společnost na konci tisícletí I, Prag: 197-209.

Mikulka, Milan (2015): Miliardáři loví další media. In: Hospodářské Noviny, 25.5.2015: 1.

Mladenovic, Ivica (2014): Basic Features of the Transition from Nominal Socialism to Political Capitalism: The Case of Serbia. In: Debatte: Journal of Contemporary Central and Eastern Europe 22(1): 5-25. 
Mujkić, Asim (2007): Mi, građani etnopolisa, Sarajevo.

Müller, Klaus (2015): Warum die Ukraine gescheitert ist. Oligarchische Konsolidierung und geopolitisches Hasardspiel. In: Prokla 45(1): 135-153.

Münchau, Wolfgang (2015): Brutal creditors have gutted the eurozone project. In: Financial Times, 14.7.2015: 9.

Musić, Goran (2013): Serbia’s Working Class in Transition 1988-2013, Belgrad.

- (2014): Serbia's Protracted Transition Under State-Led and Neoliberal Models of Capitalist Development. In: METU Studies in Development 41(3): 371-388.

Myant, Martin (2003): The Rise and Fall of Czech Capitalism. Economic Development in the Czech Republic since 1989, Cheltenham.

-/Drahokoupil, Jan (2011): Transition Economies: Political Economy in Russia, Eastern Europe and Central Asia, Hoboken.

Nekipelov, Alexander (2012): L'instauration d'une économie de marché. In: Sapir, Jacques (Hg.): La transition russe, vingt ans après, Paris: 67-118.

Nikčević, Tamara (2012): Intervju - Aleksandar Vučić. Mišković i Beko nisu sastavljali vladu. In: Vreme, 12.7.2012. URL: www.vreme.com/cms/view.php?id=1062367\&print=yes, Zugriff: 14.7.2012.

Oberndorfer, Lukas (2012a): Hegemoniekrise in Europa - Auf dem Weg zu einem autoritären Wettbewerbsetatismus? In: Forschungsgruppe Staatsprojekt Europa (Hg.): Die EU in der Krise, Münster: 50-72.

- (2012b): Die Renaissance des autoritären Liberalismus? Carl Schmitt und der deutsche Neoliberalismus. In: Prokla 42(3): 413-431.

OSW (2015): Kryzys gospodarczo-finansowy w Rosji, Raport OSW 2/2015, Warschau.

Parafianowicz, Zbigniew/Potocki, Michał (2015): Wilky żyja poza prawem. Jak Janukowycz przegrał Ukrainę, Wołowiec.

Paulsen, Sönke (2014): Bürgerkrieg der Oligarchen. In: Freitag, 18.12.2014: 9.

Pergler, Tomáš (2014): Babiš. Př́iběh oligarchy, Prag.

Pirani, Simon (2010): Change in Putin's Russia. Power, Money and People, London-New York.

Plank, Christina (2014): Die „inneren” Dimensionen des Ukraine-Konflikts. In: Kurswechsel, Nr. 3: 83-88.

Pogorzelski, Piotr (2015a): Grupa tracąca władzę. In: Nowa Europa Wschodnia, Nr. 3-4: 62-70.

- (2015b): Po drugiej stronie lustra. In: Nowa Europa Wschodnia, Nr. 1: 26-32.

Poulantzas, Nicos (2002): Staatstheorie. Politischer Überbau, Ideologie, Autoritärer Etatismus, Hamburg.

Ranković, Miloš (2013): Reformisani, pa na čelo Vlade. In: Novi Plamen 6(17): 25-28.

Rudić, Mirko (2015): Intevju: Radomir Lazović - aktivista pokreta Ne da(vi)mo Beograd: Ko nam nudi patku. In: Vreme, 9.4.2015: 16-20.

Rybář, Marek/Spáč, Peter/Voda, Petr (2014): Prezidentské vol'by na Slovensku v roku 2014,Brno

Samary, Catherine (2005): Yougoslavie de la dé composition aux enjeux européens, Paris.

Schuller, Konrad (2015): Der gestürzte Oligarch und der Rechte Sektor, in: Frankfurter Allgemeine Zeitung, 26.3.2015: 5 .

Segert, Dieter (2015): Die Ukraine - ein gespaltenes Land. In: Welttrends, Nr. 102: 21-27.

Stojiljković, Zoran (2013): Država korupcija, Belgrad.

- (2014): Šta se dogidilo Srbiji na izborima 2014. Godine. In: Novi Plamen 7(20-22): 25-26.

Studenna-Skrukwa, Marta (2014): Ukraiński Donbas. Oblicza tożamości, regionalnej, Poznań.

Stykow, Petra (2007): Marktreform und ererbtes Beziehungskapital: Unternehmer in Russland.

In: Segert, Dieter (Hg.): Postsozialismus. Hinterlassenschaften des Staatssozialismus und neue

Kapitalismen in Europa, Wien: 45-61. 
Tabery, Erik (2015): Média v éře Anreje Babiše. In: Respekt, 13.4.2015: 44-50.

Uherek Pavel (2013): „Kdo neskáče, nei Čech” aneb o nebezbečné štítivosti vůči politice. In: Listy 43(6): 5-10.

Uvalic, Milica (2010): Serbia's Transition. Towards a Better Future, Basingstoke-New York. van der Pijl, Kees (2015): Kommt die globale autoritäre Oligarchie? Annäherungen und Konflikte zwischen dem Westen und dem Rest der Welt. In: Peripherie 35(137): 47-73.

Vauchez, Antoine (2014): Démocratiser l'Europe, Paris.

Vilenica, Ana et al. (2015): STOP investorskom urbanizmu. Report from Belgrade Waterfront. In: dérive - Zeitschrift für Stadtforschung, Nr. 59: 4-9.

Yurchenko, Yuliya (2012): „Black Holes“ in the Political Economy of Ukraine: The Neoliberalization of Europe's „Wild East“. In: Debatte: Journal of Contemporary Central and Eastern Europe, 20(2-3): 125-149.

Zgodić, Esad (2011): Bosanska partitokracija, in: Časopis za kritiko znanosti 39(243): 91-104.

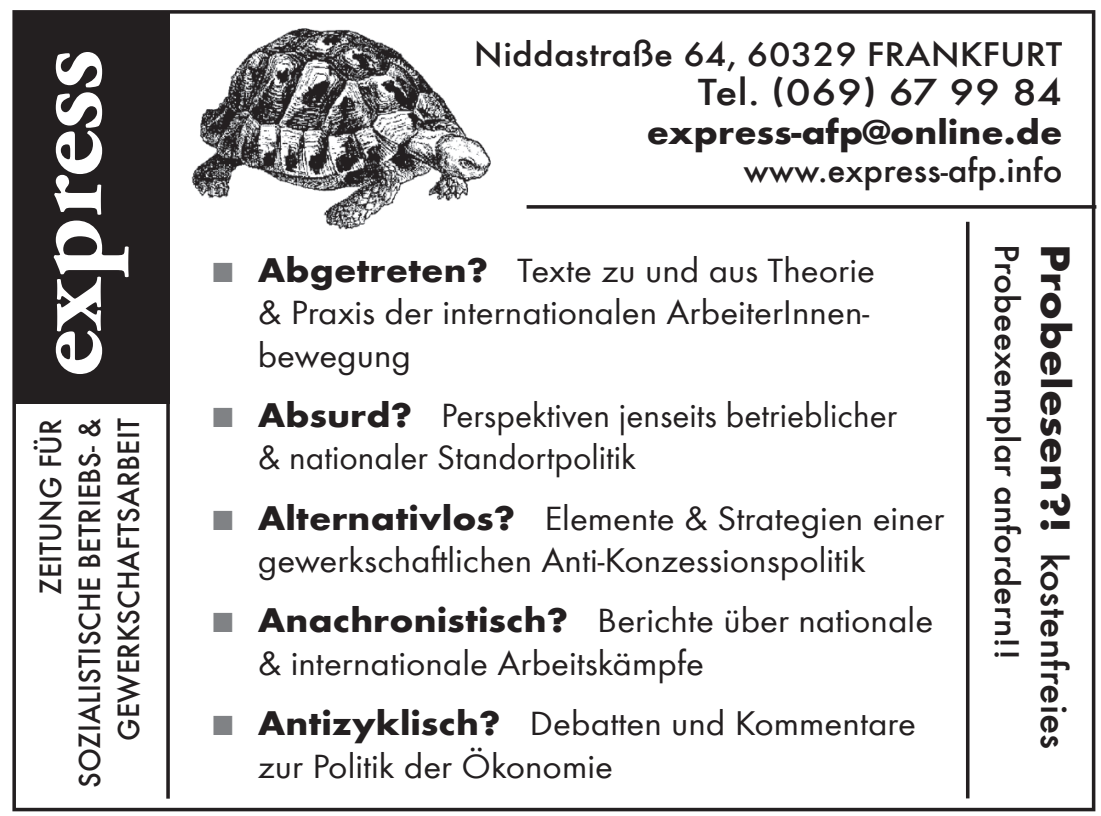

